\title{
Utility of Perioperative Warming for the Prevention of Surgical Site Infection and Patient Rehabilitative Complications: A Systematic Review
}

\author{
Authors: \\ *Aaron C. Shang, ${ }^{1,2,3}$ Kristen E. Galow, ${ }^{3}$ Jeffrey T. Essuman ${ }^{4}$ \\ 1. University of Oxford Medical Sciences Division, Oxford, UK \\ 2. University of Manchester Faculty of Biology, Medicine and Health, Manchester, UK \\ 3. Hackensack Meridian School of Medicine, Nutley, New Jersey, USA \\ 4. NHS Northeast London Commissioning Support Unit, London, UK \\ *Correspondence to aaron.shang@kellogg.ox.ac.uk
}

Disclosure: $\quad$ The authors have declared no conflicts of interest.

Received: $\quad 12.08 .2019$

Accepted: $\quad 10.12 .2019$

Keywords: $\quad$ Biostatistics, epidemiology, hypothermia, normothermia, outcomes, patients, rehabilitation, surgical site infection (SSI), surgery, warming.

Citation:

EMJ Innov. 2020;4[1]:63-72.

\section{Abstract}

Overview: Surgical site infection (SSI) is a leading cause of postoperative complication, rehospitalisation, and patient mortality after invasive clinical interventions. Surgical risks compounded by SSI introduce greater medical, economic, and quality-of-life challenges for both patients and providers alike, and to better inform clinical practice, empirical evaluation of modern surgical warming techniques is relevant. This systematic review and meta-analysis qualitatively examined the efficacy of both active and passive perioperative warming interventions upon SSI presentation versus standardised (i.e., non-warming) care.

Methods: This review analysed available literature on active and passive warming application across general anaesthesia procedures, containing longitudinal data on patient outcomes and SSI. The primary outcome studied was occurrence of post-surgical SSI; secondary outcomes included rehabilitative length of stay, attributable SSI-related mortality, and incidence of re-admittance.

Results: Meta-analysis demonstrated a significantly reduced risk ratio for SSI in patients receiving any surgical warming intervention (odds ratio: 0.36 ; 95\% confidence interval: $0.18-0.87 ; p<0.01$ ) compared to individuals treated under standard care conditions, with limited further data supporting improved active warming effect in contrast with passive implementation. Secondary postoperative outcomes, including length of rehabilitative stay or wound healing score (ASEPSIS), correspondingly demonstrated greater outcomes for surgical patients receiving perioperative warming. Introduction of warming interventions consistently correlated with reduced patient-reported pain experiences $(p<0.05)$ and downstream care expenditures $(p<0.01)$.

Conclusion: The present review identified evidence supporting a statistically significant correlation between both active and passive perioperative warming interventions to SSI prevention. These findings strongly support the recommendation of standardised perioperative warming implementation with continued investigation of relative efficacy contrasting active and passive methodologies, and across more diverse and substantial patient population sizes. 


\section{INTRODUCTION}

In modern surgical procedures, a consistent risk is the development of surgical site infection (SSI) postoperatively, projected to be affecting approximately $1-3 \%$ of all American and European patients $^{1}$ and associated with $\$ 4.25$ billion in preventable costs. ${ }^{2} \mathrm{SSI}$ are defined by the World Health Organization (WHO) and U.S. Centers for Disease Control and Prevention (CDC) as infections occurring within the physiological locale of preceding surgical intervention, typically within 30 days of operation. ${ }^{3}$ Clinical definition may include physical manifestation of discharge or swab with $>1.0 \times 10^{6}$ colony forming units per $\mathrm{mm}^{3}$ tissue, associated with at least 1 symptomology of pain, inflammation, oedema, redness, or elevated dermal temperature. ${ }^{4}$ Traditionally established risk factors for SSI presentation include the scale and scope of intervention, comorbidities, patient demographics, immunocompromisation, and diabetes, ${ }^{5}$ however, increasing recognition is becoming directed toward the role of perioperative temperature regulation (approximate euthermia 36.5-37.5 ${ }^{\circ} \mathrm{C}$ ) in maintaining patient SSI-protective immunohomeostasis. ${ }^{5}$ During surgeries, heat loss is often most significant in relation to anaesthesia introduction and general patient environmental exposure, which, in turn, downregulates immune function and instigates risk for coagulopathy, decreased basal metabolic rate, lower oxygen consumption, and immunosuppression. ${ }^{5-7}$

Operative hypothermia (core temperature $<36.5{ }^{\circ} \mathrm{C}$ ) is exceedingly common however, because of the dynamic nature of operating conditions and external factors which must be controlled for during invasive procedures. ${ }^{8}$ Literature has demonstrated that many prevalent surgical complications encompassing excessive bleeding, cardiac arrest and myocardial infarction, musculoskeletal ischaemia, and increases in all-cause rehabilitative hospital stay, as well as total expenditures for both patient and care organisations, ${ }^{9,10}$ link strongly to the persistence of surgical hypothermia. Methodologies aiming to maintain ideal patient temperatures intraoperatively have therefore become increasingly implemented, ranging from active devices, such as forced warm airflow, to passive introduction, such as heat-retaining blankets. In

isolation, these techniques have been established as efficacious for reducing intraoperative hypothermia across environmental conditions of the operating theatre. ${ }^{11,12}$ As a result, considerable assumptions regarding their intuitively protective influences against the risks of both SSI and their complications are made. ${ }^{13,14}$ In practice, however, the proposed degree of secondary generalisability amongst surgical warmingreduced normothermia and SSI reduction remains in need of robust empirical support.

Alongside relatively lacking clinical literature relating warming interventions directly against SSI presentation and related patient recovery measures, existing studies have also yielded conflicting conclusions. Most recently in 2015, Ousey et al. $^{15}$ concluded that while both active and passive perisurgical warming conferred significant benefits toward some reduction in short-term inpatient SSI recordings, follow-up tracking data suggested that these benefits disappeared when evaluated by more longitudinal factors such as SSI-related hospitalisations, further acute exacerbation, or long-term morbidity and mortality of attributable cause. Contemporary research from paediatric spine procedures indicate that surgical warming interventions significantly reduce postoperative bleeding and blood transfusion volume but generally fail to produce meaningful decreases in patient-centred results, including SSI severity and length of admittance. ${ }^{16}$ Critics of these and comparable studies have argued that small sample size, narrow surgical specialisation patient populations, and lack of effective midto-long-term follow-up measurements (beyond 1 year post surgery) often leaves negative or inconclusive findings underdeveloped.17-19 Other studies have attributed unexpectedly differing postoperative outcomes to individual factors such as patient health status ${ }^{20}$ or adherence to perioperative instruction. ${ }^{21}$ Nonetheless, they recommend that warming methods be clinically standardised to work towards reduction of SSIrelated complications by way of normothermia assurance. Throughout a seemingly contradictory assembly of conclusions on the proposed overall inter-relationship between warming, thermal conditions, and SSI development status, investigators have consistently noted potential differences between specific warming intervention methods and outcomes. Of 
particular interest is the operational divide between active and passive intervention, with some evidence to suggest that the former may confer greater effect; this debate remains actively investigated. ${ }^{22}$ At present, consultants routinely cite insufficient evidence-based findings as explanation for a widespread lack of agreement upon differential application of any or either perioperative warming approach. ${ }^{23-26}$

The present review is therefore particularly relevant for quality-of-care improvement efforts, in that quantitative evaluation of available data, associating various pathways of warming intervention with outcome variables, will inform the strongest candidates for efficacious SSI prevention. Occurrence of SSI rehospitalisation and significant postoperative complications remains a singular source of medico-economic burden, with remediation a foremost priority. Findings derived from the present study may prove especially productive for eventual construction of specialty-selective groundwork for adaptable and patient-personalised care guidelines, which may govern warming intervention within traditionally hazardous surgical theatres.

\section{METHODS}

This systematic review examines emerging clinical dissonance upon whether intraoperative clinical warming implementation, in addition to component divisions upon passive or active methodology, confer measurable benefit towards the prevention of downstream patient SSI development through alleviation of perioperative hypothermic states. Design and implementation of this study was collectively modelled upon the most recently updated version of the Preferred Reporting of Systematic Reviews and MetaAnalyses (PRISMA) framework, with direct fulfilment of the standardised 27-item checklist. ${ }^{27}$ This encompassing generalisable review and meta-analysis examines study formation (i.e., incorporation of clear inclusion and exclusion eligibility criteria) to practical application (i.e., funding disclosures).

Consequently, all included studies obtained via a comprehensive, sequential-scope, and keyword-based online literature search of PubMed/Medline/EuropePMC, ScienceDirect, and Google Scholar were screened initially by title and abstract for eligibility. Keyword-driven search parameters proceeded with increasing specificity, from generalised papers covering perioperative normothermia, to noted trends in SSI incidence, warming intervention introduction and variation, and measurable patient-specific rehabilitative indicators. Keyword implementation and database-output filtering was achieved primarily by dynamic boolean operators, followed by manual inspection of produced literature for specificity and validity. After eliminating abstracts not matching PRISMA guideline-driven selection criteria, a full-text review highlighted nine papers suitable for in-depth analyses (see Table 1 for detailed review summation).

A most recent (2018) version of the Critical Appraisal Skills Program (CASP) framework was used as the foundational guideline for determining selected studies' quality, relevance to the present review, and rigour of extracted data. In addition to CASP, specific risk of bias in selected studies were also subjected to a peer Cochrane Collaboration tool for bias assessment; this dual and apparent overlapping usage of review guidelines derives from its previously demonstrated success for increasing descriptive detail and source selection standardisation. ${ }^{28}$ Independent sensitivity testing for potential meta-analysis results confounded source material language-of-publication (consideration of non-English materials), study analysis by an alternative fixed-effects statistical model, and subjective review of related case-report and case-series literature (individual patients, or small and homogenous patient populations). All sensitivity analyses failed to significantly alter the present review's quantitative findings or ensuing qualitative discussion emphases, and therefore the overall methodology and recorded outcomes of the study are suggested as being robust (collective changes in empirical outcomes: $p>0.68$ ).

\section{Data Analysis}

Intra-study effect size between warming intervention, both actively and passively delineated, and standard non-warming care upon SSI prevalence were the primary outcomes examined. Across selected literature, standardised units of measurement were the 
recorded downstream patient occurrence SSI presentations by both raw constituency within total population (cases per total patients), as well as percentage composition. Secondary outcome measures such as morbidity and mortality are discussed by empirical proportion, and subjectively as needed.

\section{Results}

Nine studies met all the criteria and were selected for analysis in this review. The data from some 3,627 demographically diverse patients across the included literature were aggregated for systematic analysis. Individual study scope and population sizes of these studies ranged from $38^{29,30}$ to 1,824 patients. ${ }^{31,32}$

\section{Evaluation of Outcomes}

All studies reported favourable effects of warming intervention after introduction of perioperative hypothermia prevention, and literature that separated active versus passive warming preliminarily identified active means as being somewhat more efficacious in the clinic. ${ }^{30,31}$ Eight of nine examined studies significantly associated warming procedures with reduced SSI occurrence, with the lone dissenting study exhibiting marginally insignificant strength of association between said factors ( $p>0.05$; Lista et al. ${ }^{25}$ descriptive following). Publication- specific secondary outcomes varied between literature and encompassed a range of factors such as patient reintervention and readmittance rates, attributable mortality, and recovery length-of-stay. These admittedly more limited indicators generally confirmed hypothesised patient post-surgical outcome improvements concurrent with implementation of perioperative warming intervention.

Mason et al. ${ }^{33}$ found in a cohort of cancer patients ( $N=246$; aged 20-87; mean BMI: 28) that aeriated active $\mathrm{CO}_{2}$ normothermia regulation significantly decreased incidence of postoperative hypothermia (odds ratio [OR]: 0.10; 95\% confidence interval [Cl]: 0.04-0.23) alongside amplified SSI risks for patients experiencing perioperative hypothermia (OR: 4.0; 95\% Cl: 1.25-12.90), relative to standard care control. With humidification of active $\mathrm{CO}_{2}$ warming, recipient patients' relative SSI risk decreased by approximately $66 \%$ in contrast to control non-warming care $(p=0.04)$. SSI prevention-related cost-effectiveness analysis estimated net downstream healthcare savings from intraoperative warming at $£ 155$ per patient through preventable SSI-related follow-up. A post-investigative incremental cost-effectiveness ratio was concurrently negative, indicating an overall dominant cost-effective determination of SSI-prevention through intraoperative warming. ${ }^{33}$

Table 1: Selection criteria.

\begin{tabular}{|c|c|c|}
\hline Inclusion criteria & Exclusion criteria (by design) & Exclusion criteria (de facto) \\
\hline $\begin{array}{l}\text { Quantitative design (randomised controlled } \\
\text { trials preferred), clinical cohort studies also } \\
\text { included }\end{array}$ & Qualitative and non-empirical studies & $\begin{array}{l}\text { Not available in Medline/ } \\
\text { PubMed, Google Scholar, or } \\
\text { ScienceDirect }\end{array}$ \\
\hline English language & Non-English language publications & Published \\
\hline Surgical site infection as outcome measure & $\begin{array}{l}\text { Did not explicitly measure surgical site } \\
\text { infection outcome }\end{array}$ & $\begin{array}{l}\text { Relatively recent (to be available } \\
\text { and/or cited electronically) }\end{array}$ \\
\hline $\begin{array}{l}\text { Warming interventions as independent } \\
\text { variable }\end{array}$ & $\begin{array}{l}\text { Did not use warming interventions as } \\
\text { independent factor(s) }\end{array}$ & \\
\hline $\begin{array}{l}\text { Meets established literature/results quality } \\
\text { standards (see results) }\end{array}$ & $\begin{array}{l}\text { Does not meet data/study quality } \\
\text { criteria }\end{array}$ & \\
\hline Studies reference primary patient data & $\begin{array}{l}\text { Secondary patient data and/or } \\
\text { reviews }\end{array}$ & \\
\hline Passes author conflict of interest checks & Author conflict of interest noted & \\
\hline $\begin{array}{l}\text { Involved general anaesthetic surgical } \\
\text { procedures }\end{array}$ & $\begin{array}{l}\text { Non-surgical and/or localised } \\
\text { anaesthesia }\end{array}$ & \\
\hline
\end{tabular}




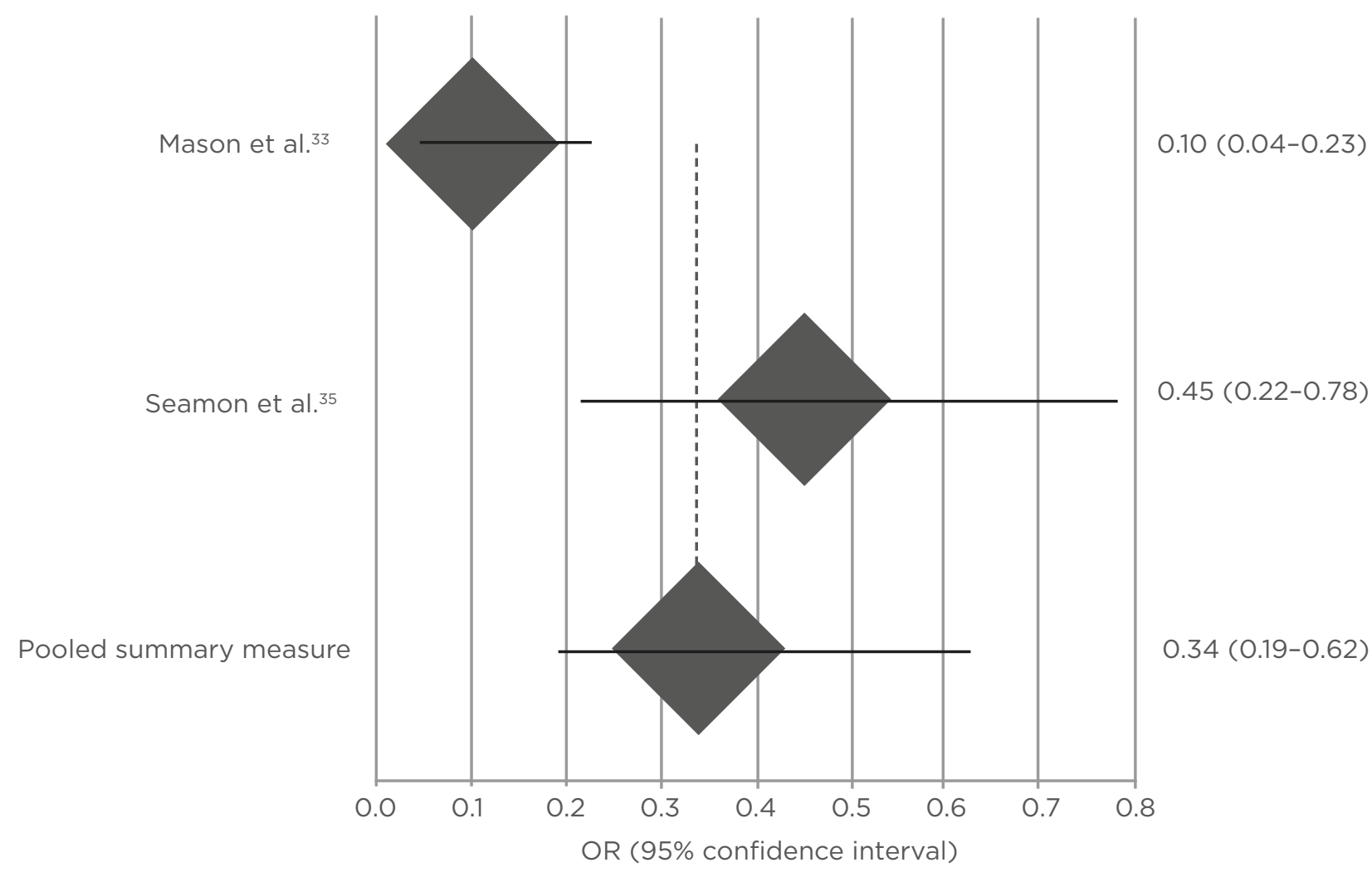

Figure 1: Meta-analysis of warming intervention and operative hypothermia by Forest plot.

Odds ratio of surgical site infection development and perioperative hypothermia [95\% confidence interval]. Odds ratio below 1.0 indicates relative risk reduction in surgical site infection for patients with warming intervention compared to standard, non-warming care.

OR: odds ratio.

Conclusive findings by Mason et al..$^{32}$ compare favourably to the study of elective open colorectal surgery, wherein $18 / 96$ patients examined experienced SSI under hypothermic surgical conditions $\left(34.7 \pm 0.6^{\circ} \mathrm{C} ; 19 \%\right)$ contrasted to $6 / 104$ patients under passive-intervention normothermia $\quad\left(36.6 \pm 0.5 \quad{ }^{\circ} \mathrm{C} ; \quad 6 \%\right) .{ }^{21}$ This statistically significant finding $(p=0.009)$ was supported by numerous secondary outcomes: faster time to suture removal for normothermia patients $(p=0.002)$ and decreased postoperative hospitalisation timeframe (2.6 days or roughly 20\%; $p=0.01$ ) compared to hypothermic condition patients. Multivariate analysis additionally identified commonly-associated risk factors of tobacco use (OR: 10.5; 95\% Cl: 3.2-34.1) and patient age (OR: 1.6; 95\% Cl: 1.0-2.4) as significant positive correlates in consequent SSI prevalence. On a much wider scale, Liau et al. ${ }^{32}$ quantified reduction in incidence of SSI from $3.1 \%$ to $0.5 \%$ among non-warming standard operative care and targeted surgical warming intervention ( $p<0.001), \quad$ a relative $84.0 \%$ decrease. Corresponding SSI prevention-associated savings were tabulated at approximately USD $\$ 147,967 .^{32}$

Melling et al. ${ }^{34}$ performed intention-to-treat analysis and identified SSI in 19/139 non-warmed hernia surgery patients (14\%) compared to 13/277 patients receiving active warming intervention (5\%; $p=0.001)$. ASEPSIS wound score severity likewise significantly decreased between warmed and control procedures $(p=0.007)$. Corresponding to reduced SSI risk for patients receiving intervention, necessity of antibiotics post-surgically was diminished relative to control $(p=0.002)$. No added significant differences were established for measurements of haematoma and seroma presentation. Follow-up in 2006 factoring in ASEPSIS standardised wound categorisation in hernia surgery again confirmed significant improvement in patients operated 
with active perioperative warming $(p<0.05)$ relative to non-warmed control, with statistically significant differences in subjective patient pain scores $(p<0.05)$, for both short ( 2 hours) and longer-term ( 7 days postoperatively) warming versus baseline. ${ }^{35}$ Similarly strong associations between warming, hypothermia reduction, and SSI prevention were shown by Wong et al. ${ }^{36}$ in generalised abdominal surgery $(\mathrm{N}=103)$. Patients operated with passive warming $(n=47)$ exhibited markedly lower SSI complication rates ( $32 \%$ versus $54 \%$ control; $p=0.027$ ) and blood loss ( $50 \%$ median quantity decrease; $p=0.011$ ) in contrast to non-warmed individuals. Anecdotally for review, this study individually reported perioperative mortality which was two amongst controls against one for within warmed patients; no relevant conclusions were drawn from this extremely incomplete dataset for warming correlation to intra-surgery mortality.

Clinical cohort analyses by Seamon et al. ${ }^{37}$ demonstrated more significant associations between intraoperative hypothermia presentation and SSI development. Within nonwarmed individuals SSI risk increased $221 \%$ per degree below $35{ }^{\circ} \mathrm{C}$, a common clinical benchmark for perioperative hypothermic states (OR: 2.21; 95\% Cl: 1.24-3.92; $p=0.007$ ). In comparison were complementary results from Lista et al. ${ }^{25}$ demonstrating significantly reduced postoperative length of stay and recovery rate over passive warming intervention $(p=0.001)$, alongside lower perioperative analgesia administration requirement $(p=0.042)$. Of note is the fact that Lista et al. ${ }^{25}$ proved the only selected study in this review that failed to identify a meaningful association between warming intervention and postoperative SSI complication incidence $(p>0.05)$. Guarded findings were observed by Smith et al. ${ }^{29,30}$ in the field of gynaecological surgery; data indicated active warming resulted in more effective perioperative hypothermia prevention than either passive normothermia maintenance or nonwarming standard surgical care, as evaluated by immediate measures and resulting occurrence of SSI (both $\mathrm{p}<0.05$ ). Longitudinal patient outcomes (SSI, morbidity, and mortality) failed to exhibit meaningful consensus. This divergence, however, was attributed to difficulties in attrition and follow-up.

\section{Meta-Analysis}

Whereas the association between warming intervention and perioperative hypothermia prevention has been assumed at times and therefore inconsistently studied in literature, currently hypothesised clinical relationships between warming intervention implementation and SSI reduction nonetheless constitutively derive from this foundation. Mason et al. ${ }^{33}$ and Seamon et al. ${ }^{37}$ determined an OR of 0.34 (95\% Cl: 0.19-0.62; Figure 1), supporting intuitive expectations that both active and passive perioperative warming confers significant control over, and effect upon, normothermia retention. Kurz et al. ${ }^{21}(p=0.009)$ and Seamon et al. $^{37} \quad(p=0.007)$ similarly indicated strong temperature regulation improvements by active warming techniques contrasted to passive, and further validated the foundational warmingto-normothermia relationship by significance stratification of SSI risk across approaches. Meta-analysis of SSI development was ORdependent, and the warming intervention demonstrated compatibly significant reductions in patient SSI post-normothermia surgeries, in contrast to procedures within which either hypothermic patient conditions were empirically identified (OR: 0.36; 95\% Cl: 0.18-0.87; $p<0.01$; Figure 2). Three selected studies in this review did not quantify SSI prevalence and warming intervention by OR measure, but nonetheless demonstrated significance between objectivelymeasured operative normothermia and reduced $\mathrm{SSI}$ presentation by counterpart risk ratio (RR) and maximum likelihood estimation $(p<0.001$; $p=0.001 ; p<0.05$; by Liau et al., ${ }^{32}$ Lista et al., ${ }^{25}$ and Smith et al., ${ }^{29}$ respectively).

Secondary outcome measures as reported by evaluated literature collectively indicated broad concurrence of perioperative improvement to SSI prevention, as well as normothermia guarantee. Relevant significant findings included time to suture removal (Kurz et al.:21 $p=0.002$ ), differences in postoperative inpatient hospitalisation time (Kurz et al..21 $p=0.01$; Wong et al..36 $p<0.05$; Seamon et al.:37 $p=0.001$ ), ASEPSIS wound score (Melling et al.:34 $p=0.007$; Melling and Leaper: ${ }^{35}$ $p<0.05$ ), patient-reported pain score (Melling and Leaper: ${ }^{35} \mathrm{p}<0.05$ ), median blood loss (Wong et al.:36 $\mathrm{p}=0.01$ ), and perioperative analgesia use (Lista et al..25 $\mathrm{p}=0.04$ ). A solitary, nonsignificant 
secondary outcome indicator, as consecutively recorded by Melling et al. ${ }^{34}$ and Lista et al., ${ }^{25}$ involved SSI-complicating haematoma and seroma frequency across surgical specialty, evaluated by both severity occurrence between warmed and non-warmed patients, whereupon interventions failed to show effective alleviation. ${ }^{35}$

\section{DISCUSSION AND IMPLICATIONS}

Against control care of non-warming operative treatments only, active warming methodologies consistently demonstrated a statistically significant effect for prevention of SSI (OR: 0.36; 95\% Cl: 0.18-0.87; $\mathrm{p}<0.01$ ) as well as improving a host of related secondary outcome measures. In comparison of active to passive implementations of warming intervention, quantifiably contrasting data was more lacking. Although, the studies that did juxtapose both approaches consistently rated active means as having magnified relevant patient warming effects, both empirically (e.g., normothermia stability, primary SSI occurrence) and subjectively (quality-of-life estimation). ${ }^{21,30,31,37}$
Applications of surgical warming intervention investigations remain far-reaching across efforts to improve clinical outcomes and constituent patient experiences. Recorded postoperative microbial infection cases in the USA in 2019 have outpaced procedurally related rehabilitative complications by many magnitudes. ${ }^{38}$ Having already been strongly correlated by prevalence with consequent SSI, ${ }^{39}$ perioperative hypothermia prevention represents a promising clinical alternative to hazardous, resource-intensive reactive therapies. Furthermore, literature strongly suggests additional medical benefit outside of simple infection-rate reduction, such as accelerated physiological neogenesis. ${ }^{40}$ Whilst no studies included for meta-analysis presently examined expectation for side-effect profiles via treatment with warming methodologies, some presentations have been noted; these include case reports of sustained burns from active forced-air warming ${ }^{30}$ owing to improper implementation, alongside ongoing debate over potential methicillin-resistant Staphylococcus aureus bacterial infection exposure risks derived. ${ }^{41}$

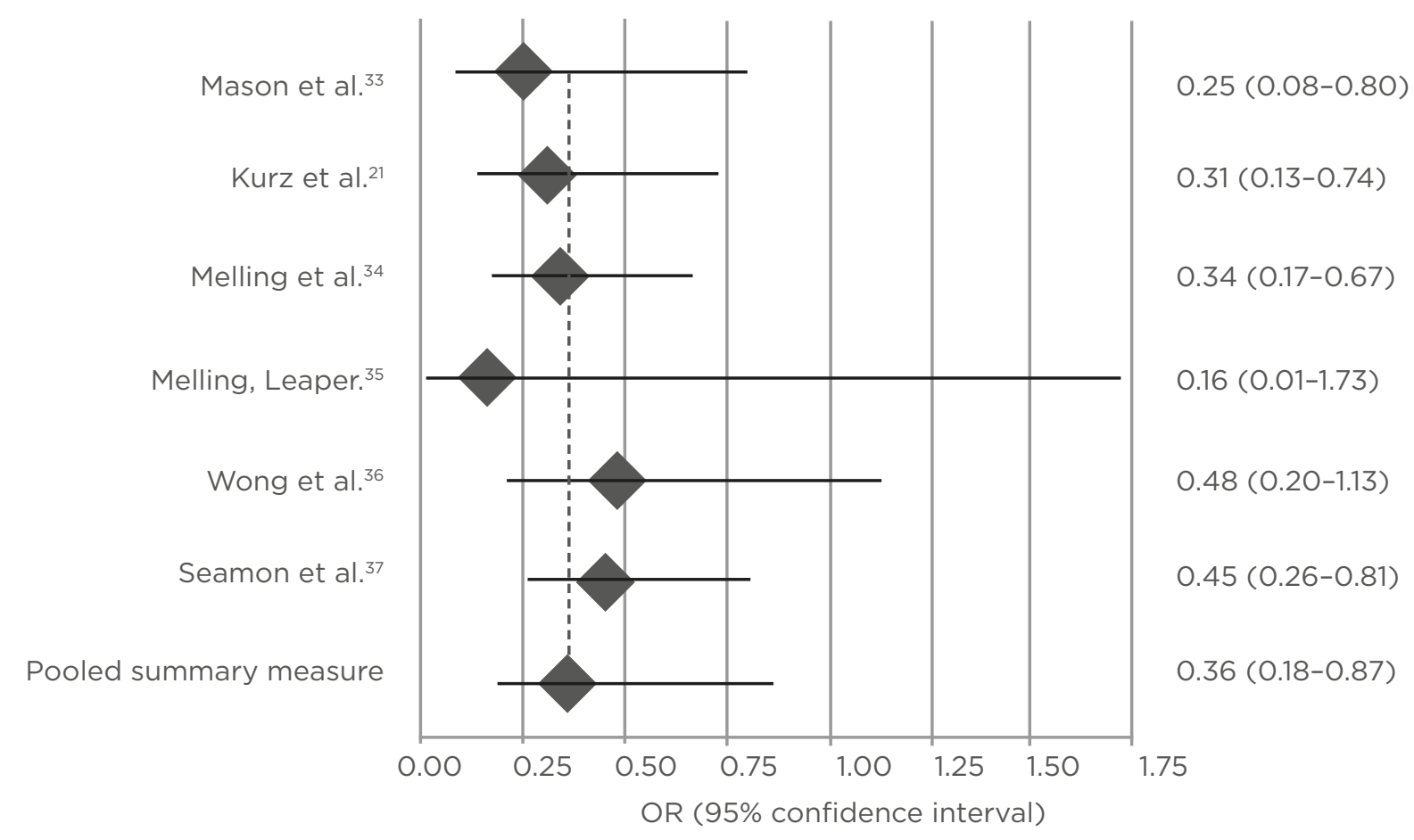

Figure 2: Meta-analysis of perioperative warming intervention and SSI risk by Forest plot.

Odds ratio of surgical site infection post-perioperative warming intervention. Odds ratio below 1.0 indicates relative risk reduction in surgical site infection for patients with warming intervention compared to standard, non-warming care.

OR: odds ratio; SSI: surgical site infection. 
Hypothesised from inherent disturbance of operating suite sterility from requisite warming techniques ranging from normothermic fluid administration to forced airflow, the validity and strength of proposed warming side-effect profiles remain actively researched. .2, $^{43}$

\section{Relevant Limitation and Biases}

Important secondary measures complementary to empirical postoperative SSI presentation rates are consistently less well-tracked in literature. Given the clinically-oriented nature of perioperative care, this presents difficulty in accurately constructing a sufficient understanding of patient postoperative outcomes in context, especially in consideration of attrition and continuity of care confounds; a greater quantity of analysable supporting measures would prove extremely valuable for confident generalisation of currently available literary findings. Procedurally, of note is some persistent, although minor, variation of institutional definition for perioperative hypothermia. Concerningly, lower normothermic boundaries varied up to $1.0{ }^{\circ} \mathrm{C}$ (35.0-36.0 ${ }^{\circ} \mathrm{C}$ ) between studies. ${ }^{11}$ Follow-up elucidation on the relevance of this dissociation and greater evidence-based operative climate benchmarks are frequently discussed in literature, yet have not been extensively investigated to date. ${ }^{44-46}$ In the present review, a slightly divergent bottom-array border differentiation between hypo and normothermic states similarly constrained confidence to generalise. Targeted research, aimed at continually elucidating patient-oriented outcome differences between hospital-determined acceptable operating suite standards, remains highly relevant for greater elucidation.

Largely inherent demographic and medical factors could have induced a degree of differential normothermia intervention, for example the complexity of surgical procedures. Prevailing clinical issues, such as morbidity and mortality, among patients' postoperative discharge due to disease state (or adherence to medical therapy, among other factors) could alter measured SSI strengths of association. Practically, extensive ethical and procedural challenges of blinding clinically-based research inevitably reduce internal validity of examined studies. ${ }^{47,48}$ Slight procedural bias could arise from this review's literature selection, as no grey literature, hand searches, or non-English sources were considered (Table 1); although, sensitivity analyses of these factors would suggest negligible collective influences upon the overall findings. Macroscopic lack of a major multicentre, prospectively randomised controlled trials constrained the availability of high-quality data. Despite such, this review remains the most updated within a modest pool of literature examining effects of perioperative warming for SSI prevention and patient rehabilitation.

\section{CONCLUSION}

Guarantee of absolute perioperative normothermia remains among the most pressing of surgical challenges. Studies across numerous surgical subspecialties suggest that important protective effects exist between perioperative hypothermia prevention and downstream reduction in SSI or related complications incidence. The current systematic review and meta-analysis empirically confirmed numerous predicted fundamental associations between warming intervention and perioperative normothermia, with significant differences in both SSI occurrence across warming and non-warming perioperative conditions, as well as some improved secondary outcome measures. However, not enough data is presently available in literature to comprehensively analyse pair-wise associations of active versus passive warming with definitive ascertainment of relative patient-safety efficacy between modalities. It should be noted that preliminary findings discussed in this review nonetheless strongly suggest that magnified active warming benefits require greater adaptability and surgical staff influence. ${ }^{49}$ The expectation therefore remains that with greater literature availability, further co-operative relationships between active surgical normothermia and SSI prevention may be strengthened across specialty and patient characterisations. Prospective investigation therein remains critical given that existing evidence is merely sufficient to endorse by clinical experience the implementation of active patient warming in the absence of exceptional patient or situational risk factors. ${ }^{50-52}$

Observed and common limitations in clinical outcomes literature, such as limited secondary 
patient-oriented postoperative rehabilitative indicators ${ }^{53-55}$ in combination with SSI occurrence, remain highly relevant and require additional subspecialty field and patient-population specific studies to elucidate for wider generalisability. Elucidation of effect size for warming interventions across wider operating methodologies, particularly in light of emerging minimally invasive surgical techniques, concurrently represent newly pressing directions of inquiry. Within retrospectively oriented research common in the clinical realm, particularly imperative is artificial elevation of Type-l error likelihood by means of structural course-oftreatment evaluation biases. ${ }^{56-58}$ Across surgical interventions in the current review, interplays between SSI prevention from novel perioperative warming relative to variations in traditional antibiotic prescriptions proved difficult to unambiguously isolate; a fine scrutiny further complicated by existing concerns on clinical prescription pattern biases regarding situational or patient characteristics that influence type or quality of care..$^{34,35,59,60}$

Considering the increasing availability of data regarding warming interventions and sustained interest in improving patient surgical outcomes, the expectation that normothermia regulation will play increasingly prominent roles across surgery is viewed as highly practical. As a largely inexpensive and easily implementable measure in modernised clinics, perioperative surgical warming should presently be routinely subscribed across surgical procedures with moderate-tostrong strength of recommendation ${ }^{61}$ in the absence of contradictory clinical findings.

\section{References}

1. Barie PS, Eachempati SR. Surgical site infections. Surg Clin. 2005;85(6):111535.

2. Umscheid, CA et al. Estimating the proportion of healthcare-associated infections that are reasonably preventable and the related mortality and costs. Infect Control Hosp Epidemiol. 2011;32(2):101-14.

3. Centers for Disease Control and Prevention (CDC). Surgical Site Infection (SSI) event, procedureassociated module. 2019. Available at: www.cdc.gov/nhsn/pdfs/ pscmanual/9pscssicurrent.pdf. Last accessed: 17 December 2019.

4. Centers for Disease Control and Prevention (CDC). Healthcareassociated infections: Surgical site infection (SSI). 2010. Available at: https://www.cdc.gov/hai/ssi/ssi.html. Last accessed: 02 August 2018.

5. Leaper DJ. Surgical-site infection. $\mathrm{Br} \mathrm{J}$ Surg. 2010;97(11):1601-2.

6. Flores-Maldonado A et al. Mild perioperative hypothermia and the risk of wound infection. Arch Med Res. 2001;32(3):227-31.

7. Harper CM. et al. Maintaining perioperative normothermia: A simple, safe, and effective way of reducing complications of surgery. BMJ. 2002;326(7392):721-2.

8. Sessler DI. Mild perioperative hypothermia. N Engl J Med. 1997;336(24):1730-7.

9. Rajagopalan $\mathrm{S}$ et al. The effects of mild perioperative hypothermia on blood loss and transfusion requirement. Anesthesiology. 2008;108(1):71-7.

10. Reynolds L et al. Perioperative complications of hypothermia. Best Pract Res Clin Anaesthesiol. 2008;22(4):645-57.

11. Torossian A. Thermal management during anaesthesia and thermoregulation standards for the prevention of inadvertent perioperative hypothermia. Best Pract Res Clin Anaesthesiol. 2008;22(4):659-68.

12. Burger L, Fitzpatrick J. Prevention of inadvertent perioperative hypothermia. $\mathrm{Br} \mathrm{J}$ Nurs. 2009;18(18):1116-9.

13. Sajid MS et al. The role of perioperative warming in surgery: A systematic review. Sao Paulo Med J. 2009:127(4):231-7.

14. Sessler DI. Perioperative thermoregulation and heat balance. Lancet. 2016;387(10038):2655-64.

15. Ousey $\mathrm{KJ}$ et al. Perioperative warming therapy for preventing surgical site infection in adults undergoing surgery. Cochrane Database Syst Rev. 2015;6:CD011731.

16. Görges $M$ et al. Preoperative warming and undesired surgical and anesthesia outcomes in pediatric spinal surgery-A retrospective cohort study. Paediatr Anaesth. 2016;26(9):866-75.

17. Forbes SS et al. Implementation of evidence-based practices for surgical site infection prophylaxis: results of a pre-and postintervention study. J Am Coll Surg. 2008;207(3):336-41.

18. Moola $\mathrm{S}$ et al. Effectiveness of strategies for the management and/ or prevention of hypothermia within the adult perioperative environment. Int J Evid Based Healthc. 2011;9(4):337-45.

19. Kellam MD et al. Forced-air warming devices and the risk of surgical site infections. AORN J. 2013;98(4):35466

20. Rowley B et al. Perioperative warming in surgical patients: A comparison of interventions. Clin Nurs Res. 2015;24(4):432-41.

21. Kurz A et al. Perioperative normothermia to reduce the incidence of surgical-wound infection and shorten hospitalization. N Engl J Med. 1996;334(19):1209-15.

22. de Brito Poveda $V$ et al. A systematic review on the effectiveness of prewarming to prevent perioperative hypothermia. J Clin Nurs. 2018;22(78):906-18

23. Brasel $\mathrm{K}$ et al. Canadian Association of General Surgeons and American College of Surgeons evidence based reviews in surgery. 21: The risk of surgical site infection is reduced with perioperative oxygen. Can J Surg. 2007;50(3):214-6.

24. Anthony $T$ et al. Evaluating an evidence-based bundle for preventing surgical site infection: A randomized trial. Arch Surg. 2011;146(3):263-9. 
25. Lista $F$ et al. The impact of perioperative warming in an outpatient aesthetic surgery setting. Aesthet Surg J. 2012;32(5):613-20.

26. Horn E-P et al. The effect of short time periods of pre-operative warming in the prevention of perioperative hypothermia. Anaesthesia. 2012;67(6):612-7.

27. Moher $\mathrm{D}$ et al. Preferred reporting items for systematic reviews and meta-analyses: The PRISMA statement. PLoS Med. 2009;6(7):e1000097.

28. Pace $\mathrm{R}$ et al. Testing the reliability and efficiency of the pilot Mixed Methods Appraisal Tool (MMAT) for systematic mixed studies review. Int J Nurs Stud. 2012;49(1):47-53.

29. Smith CE et al. Warming intravenous fluids reduces perioperative hypothermia in women undergoing ambulatory gynecological surgery. Anesth Analg. 1998;87(1):37-41.

30. Smith CE et al. Preventing hypothermia: Convective and intravenous fluid warming versus convective warming alone. J Clin Anesth. 1998;10(5):380-5.

31. De Witte JL et al. Resistive-heating or forced-air warming for the prevention of redistribution hypothermia. Anesth Analg. 2010;110(3):829-33.

32. Liau K-H et al. Outcome of a strategy to reduce surgical site infection in a tertiary-care hospital. Surg Infect (Larchmt). 2010;11(2):151-9.

33. Mason SE et al. Postoperative hypothermia and surgical site infection following peritoneal insufflation with warm, humidified carbon dioxide during laparoscopic colorectal surgery: A cohort study with cost-effectiveness analysis. Surg Endosc. 2017;31(4):1923-9.

34. Melling $A C$ et al. Effects of preoperative warming on the incidence of wound infection after clean surgery: A randomised controlled trial. Lancet. 2001;358(9285):876-80.

35. Melling AC, Leaper DJ. The impact of warming on pain and wound healing after hernia surgery: A preliminary study. J Wound Care. 2006;15(3):104-

36. Wong PF et al. Randomized clinical trial of perioperative systemic warming in major elective abdominal surgery. Br J Surg. 2007;94(4):421-6.

37. Seamon MJ et al. The effects of intraoperative hypothermia on surgical site infection: An analysis of
524 trauma laparotomies. Ann Surg. 2012;255(4):789-95.

38. Brandt $\mathrm{C}$ et al. Operating room ventilation with laminar airflow shows no protective effect on the surgical site infection rate in orthopedic and abdominal surgery. Ann Surg. 2008;248(5):695-700

39. Neumayer $L$ et al. Multivariable predictors of postoperative surgical site infection after general and vascular surgery: Results from the patient safety in surgery study. J Am Coll Surg. 2007;204(6):1178-87

40. Bernard $\mathrm{H}$. Patient warming in surgery and the enhanced recovery. Br J Nurs. 2013;22(6):319-20.

41. Greif R et al. Supplemental perioperative oxygen to reduce the incidence of surgical-wound infection N Engl J Med. 2000;342(3):161-7.

42. Reed $M$ et al. Forced air warming design: Evaluation of intake filtration, internal microbial buildup, and airborne-contamination emissions. AANA J. 2013;81(4):275-80

43. Moretti $\mathrm{B}$ et al. Active warming systems to maintain perioperative normothermia in hip replacement surgery: A therapeutic aid or a vector of infection? J Hosp Infect. 2009;73(1):58-63.

44. Andrzejowski JL et al. Effect of prewarming on post-induction core temperature and the incidence of inadvertent perioperative hypothermia in patients undergoing general anaesthesia. $\mathrm{Br} J$ Anaesth. 2008:101(5):627-31.

45. Campbell $\mathrm{G}$ et al. Warming of intravenous and irrigation fluids for preventing inadvertent perioperative hypothermia. Cochrane Database Syst Rev. 2015:2015(4):CD009891.

46. Giesbrecht GG et al. Comparison of forced-air patient warming systems for perioperative use. Anesthesiology. $1994: 80(3): 671-9$

47. Godwin M et al. Pragmatic controlled clinical trials in primary care: The struggle between external and internal validity. BMC Med Res Methodol. 2003;3:28

48. Rothwell PM et al. External validity of randomised controlled trials: "To whom do the results of this trial apply?" Lancet. 2005;365(9453):8293.

49. Negishi $C$ et al. Resistive-heating and forced-air warming are comparably effective. Anesth Analg. 2003;96(6):1683-7.
50. Chiang $\mathrm{N}$ et al. Perioperative warming, oxygen, and llomedin on oxygenation and healing in infrainguinal bypass surgery. J Surg Res. 2017;220:197-205.

51. Leslie K, Sessler DI. Perioperative hypothermia in the high-risk surgical patient. Best Pract Res Clin Anaesthesiol. 2003;17(4):485-98.

52. Madrid E et al. Active body surface warming systems for preventing complications caused by inadvertent perioperative hypothermia in adults. Cochrane Database Syst Rev. 2016;2016(4):CD009016.

53. Constantine RS et al. The impact of perioperative hypothermia on plastic surgery outcomes: A multivariate logistic regression of 1062 cases. Aesthet Surg J. 2015;35(1):81-8.

54. Fettes $S$ et al. Effect of preoperative forced-air warming on postoperative temperature and postanesthesia care unit length of stay. AORN J. 2013;97(3):323-8

55. Steelman VM et al. Warming of irrigation fluids for prevention of perioperative hypothermia during arthroscopy: A systematic review and meta-analysis. Arthroscopy. 2018;34(3):930-42.e2

56. Poolman RW et al. Reporting of outcomes in orthopaedic randomized trials: Does blinding of outcome assessors matter? J Bone Joint Surg Am. 2007;89(3):550-8.

57. Schulz KF et al. Empirical evidence of bias: Dimensions of methodological quality associated with estimates of treatment effects in controlled trials. JAMA. 1995:273(5):408-12.

58. Wood L et al. Empirical evidence of bias in treatment effect estimates in controlled trials with different interventions and outcomes: Meta-epidemiological study. BMJ. 2008;336:601.

59. Chilet-Rosell E. Gender bias in clinical research, pharmaceutical marketing, and the prescription of drugs. Glob Health Action. 2014;7.doi:10.3402/gha. v7.25484.

60. Petri $\mathrm{H}$, Urquhart J. Channeling bias in the interpretation of drug effects. Stat Med. 1991;10(4):577-81.

61. Ebell $\mathrm{MH}$ et al. Strength of recommendation taxonomy (SORT): A patient-centered approach to grading evidence in the medical literature. J Am Board Fam Med. 2004;17(1):59-6 